\title{
An Adaptive Super-Exponential Deflation Algorithm for Blind Deconvolution of MIMO Systems Using the Matrix Pseudo-Inversion Lemma
}

\author{
Kiyotaka Kohno*, Yujiro Inouye $^{\dagger}$ and Mitsuru Kawamoto ${ }^{\ddagger}$ \\ ${ }^{*} \dagger \ddagger$ Department of Electronic and Control Systems Engineering, Shimane University \\ 1060 Nishikawatsu, Matsue, Shimane 690-8504, Japan \\ *kohno@yonago-k.ac.jp, ${ }^{\dagger}$ inouye@ $@$ riko.shimane-u.ac.jp, ${ }^{\ddagger}$ kawa@ecs.shimane-u.ac.jp
}

\begin{abstract}
The multichannel blind deconvolution of finiteimpulse response (FIR) or infinite-impulse response (IIR) channels is investigated using the multichannel super-exponential deflation methods. We propose a new adaptive approach to the multichannel super-exponential deflation methods using the matrix pseudo-inversion lemma (which is extended from the matrix inversion lemma in the full-rank case to the rank-degenerate case) and the higher-order cross correlations of the channel and the equalizer outputs. In order to see the effectiveness of the proposed approach, many computer simulations are carried out for time-invariant MIMO channels along with time-variant MIMO channels. It is shown through computer simulations that the proposed approach is effective for even time-variant channels.
\end{abstract}

\section{INTRODUCTION}

Multichannel blind deconvolution has recently received attention in such fields as digital communications, image processing and neural information processing [1].

In the early 1990s, Shalvi and Weinstein proposed an attractive approach to single-channel blind deconvolution called the super-exponential methods (SEMs) [2]. One of the attractive properties of the SEM is that it converges iteratively to desired solution regardless of initialization at a superexponential rate. Extensions of their idea to multichannel deconvolution were presented in [3], [4], [5], [6], [7], [9]. In particular, Inouye and Tanebe [3] proposed the multichannel super-exponential deflation method (MSEDM) using the second-order correlations. Moreover, Kawamoto et al. [7] and Kohno et al. [9] proposed MSEDMs using the higher-order correlations instead of the second-order correlations in order to reduce the computational complexity and accelerate the performance of deconvolution. The MSEDMs are to deconvolve sequentially the source signals one by one. The most important property of the MSEDMs is that it converges globally to desired solution except for pathological cases. However, it is not considered that the underlying channel exhibits change in time for almost all the conventional MSEDMs, because they do not have an adaptive algorithm which is capable of tracking the varying characteristics of the channel.

In the present paper, we propose an adaptive multichannel super-exponential deflation algorithm (AMSEDA) using the higher-order correlations for MIMO wide band channels (convolutive mixtures). We already proposed two type of adaptive multichannel super-exponential algorithms (AMSEAs), the one in covariance (correlation or Kalman-filter) form and the other in QR-factorization form, for the degenerate rank case of the correlations matrices [8]. We propose an AMSEDA using the matrix pseudo-inversion lemma (the covariance form) in this paper, and we show the effectiveness of the proposed algorithm by computer simulations in comparison with the AMSEDA using the QR-factorization [10].

The present paper uses the following notation: Let $Z$ denote the set of all integers. Let $\boldsymbol{C}^{m \times n}$ denote the set of all $m \times n$ matrices with complex components. The superscripts $T, *$, $H$ and $\dagger$ denote, respectively, the transpose, the complex conjugate, the complex conjugate transpose (Hermitian) and the (Moore-Penrose) pseudoinverse operations of a matrix. Let $i=\overline{1, n}$ stand for $i=1,2, \cdots, n$.

\section{Assumptions And Preliminaries}

We consider an MIMO channel with $n$ inputs and $m$ outputs as described by

$$
\boldsymbol{y}(t)=\sum_{k=-\infty}^{\infty} \boldsymbol{H}^{(k)} \boldsymbol{s}(t-k), \quad t \in Z
$$

where

$s(t) \quad n$-column vector of input (or source) signals,

$\boldsymbol{y}(t) \quad m$-column vector of channel outputs,

$\boldsymbol{H}^{(k)} \quad m \times n$ matrix of impulse responses.

The transfer function of the channel is defined by

$$
\boldsymbol{H}(z)=\sum_{k=-\infty}^{\infty} \boldsymbol{H}^{(k)} z^{k}, \quad z \in C .
$$

For the time being, it is assumed for theoretical analysis that the noise is absent in (1).

To recover the source signals, we process the output signals by an $n \times m$ equalizer (or deconvolver) $\boldsymbol{W}(z)$ described by

$$
\boldsymbol{z}(t)=\sum_{k=-\infty}^{\infty} \boldsymbol{W}^{(k)} \boldsymbol{y}(t-k), \quad t \in Z
$$

The objective of multichannel blind deconvolution is to construct an equalizer that recovers the original source signals only from the measurements of the corresponding outputs.

We put the following assumptions on the channel and the source signals.

A1) The transfer function $\boldsymbol{H}(z)$ is stable and has full column rank on the unit circle $|z|=1$ [ this implies that the unknown 
system has less inputs than outputs, i.e., $n \leq m$, and there exists a left stable inverse of the unknown system ].

A2) The input sequence $\{s(t)\}$ is a complex, zero-mean, non-Gaussian random vector process with element processes $\left\{s_{i}(t)\right\}, i=\overline{1, n}$ being mutually independent. Moreover, each element process $\left\{s_{i}(t)\right\}$ is an i.i.d. process with a nonzero variance $\sigma_{i}^{2}$ and a nonzero fourth-order cumulant $\gamma_{i}$. The variances $\sigma_{i}^{2}$ 's and the fourth-order cumulants $\gamma_{i}$ 's are unknown. A3) The equalizer $\boldsymbol{W}(z)$ is an FIR channel of sufficient length $L$ so that the truncation effect can be ignored.

Remark 1: As to A1), if the channel $\boldsymbol{H}(z)$ is FIR, then a condition of the existence of an FIR equalizer is rank $\boldsymbol{H}(z)=$ $n$ for all nonzero $z \in C$ [11]. Moreover, if $\boldsymbol{H}(z)$ is irreducible, then there exists an equalizer $\boldsymbol{W}(z)$ of length $L<n(K-1)$, where $K$ is the length of the channel [11]. Besides, it is shown in [4] that there exists generically (or except for pathological cases) an equalizer $\boldsymbol{W}(z)$ of length $L=\left\lceil\frac{n(K-1)}{m-n}\right\rceil$, where $\lceil x\rceil$ stands for the smallest integer that is greater than equal to $x$.

Let us consider an FIR equalizer with the transfer function $\boldsymbol{W}(z)$ given by

$$
\boldsymbol{W}(z)=\sum_{k=L_{1}}^{L_{2}} \boldsymbol{W}^{(k)} z^{k},
$$

where the length $L:=L_{2}-L_{1}+1$ is taken to be sufficiently large. Let $\tilde{\boldsymbol{w}}_{i}$ be the $L m$-column vector consisting of the tap coefficients (corresponding to the $i$ th output) of the equalizer defined by

$$
\begin{aligned}
& \tilde{\boldsymbol{w}}_{i}:=\left[\boldsymbol{w}_{i, 1}^{T}, \boldsymbol{w}_{i, 2}^{T}, \cdots, \boldsymbol{w}_{i, m}^{T}\right]^{T} \in \boldsymbol{C}^{m L}, \\
& \boldsymbol{w}_{i, j}=\left[w_{i, j}{ }^{\left(L_{1}\right)}, w_{i, j}{ }^{\left(L_{1}+1\right)}, \cdots, w_{i, j}{ }^{\left(L_{2}\right)}\right]^{T} \in \boldsymbol{C}^{L},
\end{aligned}
$$

where $w_{i, j}{ }^{(k)}$ is the $(i, j)$ th element of matrix $\boldsymbol{W}^{(k)}$.

Inouye and Tanebe [3] proposed the multichannel superexponential algorithm (MSEA) for finding the tap coefficient vectors $\tilde{\boldsymbol{w}}_{i}$ 's of the equalizer $\boldsymbol{W}(z)$, of which each iteration consists of the following two steps:

$$
\begin{array}{ll}
\tilde{\boldsymbol{w}}_{i}^{[1]}=\tilde{\boldsymbol{R}}_{L}^{\dagger} \tilde{\boldsymbol{d}}_{i} \quad \text { for } i=\overline{1, n}, \\
\tilde{\boldsymbol{w}}_{i}^{[2]}=\frac{\tilde{\boldsymbol{w}}_{i}^{[1]}}{\sqrt{\tilde{\boldsymbol{w}}_{i}^{[1] H} \tilde{\boldsymbol{R}}_{L} \tilde{\boldsymbol{w}}_{i}^{[1]}}} \text { for } i=\overline{1, n},
\end{array}
$$

where $(\cdot)^{[1]}$ and $(\cdot)^{[2]}$ stand respectively for the result of the first step and the result of the second step. Let $\tilde{\boldsymbol{y}}(t)$ be the $L m$-column vector consisting of the $L$ consecutive inputs of the equalizer defined by

$$
\begin{array}{r}
\tilde{\boldsymbol{y}}(t):=\left[\overline{\boldsymbol{y}}_{1}(t)^{T}, \overline{\boldsymbol{y}}_{2}(t)^{T}, \cdots, \overline{\boldsymbol{y}}_{m}(t)^{T}\right]^{T} \in \boldsymbol{C}^{m L}, \\
\overline{\boldsymbol{y}}_{i}(t):=\left[y_{i}\left(t-L_{1}\right), y_{i}\left(t-L_{1}-1\right), \cdots, y_{i}\left(t-L_{2}\right)\right]^{T} \\
\in \boldsymbol{C}^{L},
\end{array}
$$

where $y_{i}(t)$ is the $i$ th element of the output vector $\boldsymbol{y}(t)$ of the channel in (1). Then the correlation matrix $\tilde{\boldsymbol{R}}_{L}$ is represented as

$$
\tilde{\boldsymbol{R}}_{L}=E\left[\tilde{\boldsymbol{y}}^{*}(t) \tilde{\boldsymbol{y}}^{T}(t)\right] \in \boldsymbol{C}^{m L \times m L},
$$

and the fourth-order cumulant vector $\tilde{\boldsymbol{d}}_{i}$ is represented as

$$
\begin{aligned}
\tilde{\boldsymbol{d}}_{i}= & \operatorname{cum}\left(z_{i}(t), z_{i}(t), z_{i}^{*}(t), \tilde{\boldsymbol{y}}^{*}(t)\right) \\
= & E\left[\left|z_{i}(t)\right|^{2} z_{i}(t) \tilde{\boldsymbol{y}}^{*}(t)\right] \\
& -2 E\left[\left|z_{i}(t)\right|^{2}\right] E\left[z_{i}(t) \tilde{\boldsymbol{y}}^{*}(t)\right]
\end{aligned}
$$

$$
-E\left[z_{i}^{2}(t)\right] E\left[z_{i}^{*}(t) \tilde{\boldsymbol{y}}^{*}(t)\right] \in \boldsymbol{C}^{m L},
$$

where $E[x]$ denotes the expectation of a random variable $x$. We note that the last term can be ignored in case of $E\left[s_{i}(t)^{2}\right]$ $=0$, in which case $E\left[z_{i}(t)^{2}\right]=0$ for all $i=\overline{1, n}$.

\section{An Adaptive Super-Exponential Algorithm \\ USING THE MATRIX PSEUDO-INVERSION LEMMA}

Kohno et al. proposed two types of AMSEAs, the one in covariance (correlation or Kalman-filter) form and the other in QR-factorization form, for the degenerate rank case of the correlations matrices [8]. Except for the case when the number of outputs equals the number of inputs, i.e., $m=n$, the correlation matrix $\tilde{\boldsymbol{R}}_{L}$ is not of full rank. Situations with the number of independent sources (or inputs) being strictly less than the number of sensors (or outputs) are often encountered in various applications such as digital communication, image processing and neural information processing. Moreover, if the underlying channel exhibits slow changes in time, processing all the available data jointly is not desirable, even if we can accommodate the computational and storage loads of the batch algorithm in (7) and (8), because different data segments correspond to different channel responses. In such a case, we want to have an adaptive algorithm which is capable of tracking the varying characteristics of the channel.

Consider the batch algorithm in (7) and (8). The equation (8) constraints a weighted norm of vector $\tilde{\boldsymbol{w}}_{i}$ to equal one, and thus we assume this constraint is always satisfied using a normalization or an automatic gain control (AGC) of $\tilde{\boldsymbol{w}}_{i}$ at each time $t$. To develop an adaptive version of (7), we must specify the dependency of each time $t$ and rewrite (7) as

$$
\tilde{\boldsymbol{w}}_{i}(t)=\tilde{\boldsymbol{R}}_{L}^{\dagger}(t) \tilde{\boldsymbol{d}}_{i}(t) \quad, \quad i=\overline{1, n} .
$$

Here the subscript $L$ of $\tilde{\boldsymbol{R}}_{L}(t)$ is omitted for simplicity hereafter.

In order to develop an adaptive version of the MSEA, we should obtain recursion formulas for time-updating of matrix $\tilde{\boldsymbol{R}}(t)$, vector $\tilde{\boldsymbol{d}}_{i}(t)$ and pseudoinverse $\tilde{\boldsymbol{R}}^{\dagger}(t)$ in (13), respectively.

$$
\begin{aligned}
\tilde{\boldsymbol{R}}(t) & =\alpha \tilde{\boldsymbol{R}}(t-1)+(1-\alpha) \tilde{\boldsymbol{y}}^{*}(t) \tilde{\boldsymbol{y}}^{T}(t), \\
\tilde{\boldsymbol{d}}_{i}(t) & =\alpha \tilde{\boldsymbol{d}}_{i}(t-1)+(1-\alpha) \tilde{\boldsymbol{y}}^{*}(t) \tilde{z}_{i}(t),
\end{aligned}
$$

where

$$
\tilde{z}_{i}(t):=\left(\left|z_{i}(t)\right|^{2}-2<\left|z_{i}(t)\right|^{2}>\right) z_{i}(t)-<z_{i}^{2}(t)>z_{i}^{*}(t) .
$$

Here $<\left|z_{i}(t)\right|^{2}>$ and $<z_{i}^{2}(t)>$ denote respectively the estimates of $E\left[\left|z_{i}(t)\right|^{2}\right]$ and $E\left[z_{i}(t)^{2}\right]$ at time $t, \alpha$ is a positive constant close to, but less than one, which accounts for some exponential weighting factor or forgetting factor [13].

By applying the pseudo-inversion lemma [8] to (14) for obtaining a recursion formula for time-updating of pseudoinverse $\boldsymbol{P}(t)=\tilde{\boldsymbol{R}}^{\dagger}(t)$, we obtain the following lemma.

Lemma 1: Let $\boldsymbol{b}(t), \boldsymbol{b}_{1}(t)$ and $\boldsymbol{b}_{2}(t)$ are defined as

$$
\begin{aligned}
& \boldsymbol{b}(t)=\sqrt{(1-\alpha)} \tilde{\boldsymbol{y}}^{*}(t), \\
& \boldsymbol{b}_{1}(t)=\boldsymbol{P}(t-1) \tilde{\boldsymbol{R}}(t-1) \boldsymbol{b}(t), \\
& \boldsymbol{b}_{2}(t)=\{\boldsymbol{I}-\boldsymbol{P}(t-1) \tilde{\boldsymbol{R}}(t-1)\} \boldsymbol{b}(t) .
\end{aligned}
$$

Then formula of the recursion for the pseudoinverse $\boldsymbol{P}(t)$ from $\boldsymbol{P}(t-1)$ by using the pseudo-inversion lemma is explicitly 
expressed, depending on the values of vectors $\boldsymbol{b}_{1}(t)$ and $\boldsymbol{b}_{2}(t)$ and matrix $\tilde{\boldsymbol{R}}(t-1)$, as follows:

1) If $\boldsymbol{b}_{2}(t)=0$, then

$$
\boldsymbol{P}(t)=\frac{1}{\alpha}\left[\boldsymbol{P}(t-1)-\frac{\boldsymbol{P}(t-1) \boldsymbol{b}_{1}(t) \boldsymbol{b}_{1}^{H}(t) \boldsymbol{P}(t-1)}{\alpha+\boldsymbol{b}_{1}^{H}(t) \boldsymbol{P}(t-1) \boldsymbol{b}_{1}(t)}\right] .
$$

2) If $\boldsymbol{b}_{2}(t) \neq 0$ and $\boldsymbol{b}_{1}(t)=0$, then

$$
\boldsymbol{P}(t)=\frac{1}{\alpha} \boldsymbol{P}(t-1)+\frac{\boldsymbol{b}_{2}(t) \boldsymbol{b}_{2}^{H}(t)}{\left\{\boldsymbol{b}_{2}^{H}(t) \boldsymbol{b}_{2}(t)\right\}^{2}} .
$$

3) Let $l(t)$ be a non-negative number defined by

$$
\begin{aligned}
l(t):= & \left|1+\boldsymbol{b}_{1}^{H}(t) \boldsymbol{P}_{b}(t) \boldsymbol{b}_{2}(t)\right|^{2} \\
& -\boldsymbol{b}_{1}^{H}(t) \boldsymbol{P}_{b}(t) \boldsymbol{b}_{1}(t) \boldsymbol{b}_{2}^{H}(t) \boldsymbol{P}_{b}(t) \boldsymbol{b}_{2}(t),
\end{aligned}
$$

where $\boldsymbol{P}_{b}(t)$ is defined by

$$
\begin{aligned}
\boldsymbol{P}_{b}(t):= & \frac{1}{\alpha}\left[\boldsymbol{P}(t-1)-\frac{\boldsymbol{P}(t-1) \boldsymbol{b}_{1}(t) \boldsymbol{b}_{1}^{H}(t) \boldsymbol{P}(t-1)}{\alpha+\boldsymbol{b}_{1}^{H}(t) \boldsymbol{P}(t-1) \boldsymbol{b}_{1}(t)}\right] \\
& +\frac{\boldsymbol{b}_{2}(t) \boldsymbol{b}_{2}^{H}(t)}{\left\{\boldsymbol{b}_{2}^{H}(t) \boldsymbol{b}_{2}(t)\right\}^{2}} .
\end{aligned}
$$

Then in the case when $\boldsymbol{b}_{2}(t) \neq 0$ and $\boldsymbol{b}_{1}(t) \neq 0$,

$\boldsymbol{P}(t)=\boldsymbol{P}_{b}(t)-\boldsymbol{P}_{b}(t)\left[\boldsymbol{b}_{1}(t), \boldsymbol{b}_{2}(t)\right] \boldsymbol{P}_{d}(t)\left[\boldsymbol{b}_{1}(t), \boldsymbol{b}_{2}(t)\right]^{H} \boldsymbol{P}_{b}(t)$,

where

$\boldsymbol{P}_{d}(t):=\frac{1}{l(t)}\left[\begin{array}{c|c}-\boldsymbol{b}_{2}^{H}(t) \boldsymbol{P}_{b}(t) \boldsymbol{b}_{2}(t) & 1+\boldsymbol{b}_{1}^{H}(t) \boldsymbol{P}_{b}(t) \boldsymbol{b}_{2}(t) \\ \hline 1+\boldsymbol{b}_{2}^{H}(t) \boldsymbol{P}_{b}(t) \boldsymbol{b}_{1}(t) & -\boldsymbol{b}_{1}^{H}(t) \boldsymbol{P}_{b}(t) \boldsymbol{b}_{1}(t)\end{array}\right]$

These equations are initialized by their values appropriately selected or calculated by the batch algorithm in (7) and (8) at initial time $t_{0}$ and used for $t=t_{0}+1, t_{0}+2, \cdots$.

Remark 2: In Lemma 1, in order to keep the stability of $\boldsymbol{P}(t)$, the positive constant value $\alpha$ has to be chosen for the appropriate value which is very close to 1 , or which is asymptotically approached to 1 with time $t$, for example $\alpha=1-1 /(1000+t)$.

Based on Lemma 1 along with (15), we obtain following theorem with gives a recursion formula for time-updating of the tap vector $\tilde{\boldsymbol{w}}_{i}(t)$ for $i=\overline{1, n}$.

Theorem 1: The recursion for $\tilde{\boldsymbol{w}}_{i}(t)$ is

$$
\tilde{\boldsymbol{w}}_{i}(t)=\boldsymbol{P}(t) \tilde{\boldsymbol{R}}(t) \tilde{\boldsymbol{w}}_{i}(t-1)+\boldsymbol{k}(t)\left[\tilde{z}_{i}(t)-\tilde{\boldsymbol{y}}^{T}(t) \tilde{\boldsymbol{w}}_{i}(t-1)\right],
$$

where

$$
\begin{aligned}
& \boldsymbol{k}(t):=(1-\alpha) \boldsymbol{P}(t) \tilde{\boldsymbol{y}}^{*}(t), \\
& \tilde{z}_{i}(t):=\left(\left|z_{i}(t)\right|^{2}-2<\left|z_{i}(t)\right|^{2}>\right) z_{i}(t)-<z_{i}^{2}(t)>z_{i}^{*}(t), \\
& <\left|z_{i}(t)\right|^{2}>:=\beta<\left|z_{i}(t-1)\right|^{2}>+(1-\beta)\left|z_{i}(t)\right|^{2}, \\
& <z_{i}^{2}(t)>:=\beta<z_{i}^{2}(t-1)>+(1-\beta) z_{i}^{2}(t) .
\end{aligned}
$$

Here $\beta$ is a positive constant less than $\alpha$, and $\boldsymbol{P}(t)=\tilde{\boldsymbol{R}}^{\dagger}(t)$ is the pseudoinverse of $\tilde{\boldsymbol{R}}(t)$.

\section{An Adaptive Super-Exponential Deflation ALgORITHM USING THE MATRIX PSEUdo-InVERSION LEMMA}

The MSEDM proposed by Inouye and Tanebe [3] uses the second-order correlations to estimate the contributions of an extracted source signal to the channel outputs. Kohno et al. [9] proposed an MSEDM using the higher-order correlations instead of the second-order correlations to reduce the computational complexity in terms of multiplications and to accelerate the performance of equalization. For the details of the MSEDM using the higher-order correlations, see the equations from (13) through (30) in [9]. In the present paper, we proposed a new AMSEDA which is an adaptive version of the MSEDM using the higher-order correlations and the matrix pseudo-inversion lemma described in the previous chapter.

In the new AMSEDA, the following procedures are carried out in each time when channel outputs are observed.

Before the following procedures are carried out, it is necessary that $\tilde{\boldsymbol{R}}, \tilde{\boldsymbol{d}}_{i}, \tilde{\boldsymbol{w}}_{i}$ and $\boldsymbol{P}$ are initialized.

At first, set $t=t_{0}$, and set $l=1$ where $l$ denotes the number of channels (or the sources) equalized.

Then, $\tilde{\boldsymbol{R}}(t)$ is calculated by (14), $\tilde{\boldsymbol{d}}_{1}(t)$ is calculated by using (15), (28), (29) and (30), $\boldsymbol{P}(t)$ is calculated by using from (17) to (25), and $\tilde{\boldsymbol{w}}_{1}(t)$ is calculated by the two steps (26) and (8). By these procedures, the first equalized output $z_{1}(t)$ is obtained.

Next, the MSEDM using the higher-order correlations is carried out. We calculate the contribution signals by using the equalized output $z_{1}(t)$, and remove the contribution signals from the channel outputs in order to define the outputs of a

\begin{tabular}{|c|c|}
\hline Step & Contents \\
\hline 1 & Set $t=t_{0}$ (where $t_{0}$ is an initial time). \\
\hline 2 & $\begin{array}{l}\text { Set } l=1 \text { (where } l \text { denotes the number of the } \\
\text { channels equalized). }\end{array}$ \\
\hline 3 & Calculate $\tilde{\boldsymbol{R}}(t)$ using (14). \\
\hline 4 & Calculate $\tilde{\boldsymbol{d}}_{l}(t)$ using (15), (28), (29) and (30). \\
\hline 5 & Calculate $\boldsymbol{P}(t)$ using from (17) to $(25)$. \\
\hline 6 & Calculate $\tilde{\boldsymbol{w}}_{l}(t)$ using (26) and (8). \\
\hline 7 & $\begin{array}{l}\text { Carry out the deflationary process using the } \\
\text { MSEDM with the higher-order correlations [9]. }\end{array}$ \\
\hline 8 & $\begin{array}{l}\text { If the subscript } l \text { is less than } n \text {, then set } \\
l=l+1 \text {, and the procedures (from Step } 3 \\
\text { through Step 7) are continued until } l=n \text {. }\end{array}$ \\
\hline 9 & $\begin{array}{l}\text { If } t<t_{f} \text { (where } t_{f} \text { is a final time), then set } \\
t=t+1 \text { and iterate the procedures from } \\
\text { Step } 2 \text { through Step } 8 \text {. If } t=t_{f} \text {, then stop here. }\end{array}$ \\
\hline
\end{tabular}
multichannel with $n-1$ inputs and $m$ outputs. The number of inputs becomes deflated by one. The procedures mentioned above are continued until $l=n$, where we obtain the last equalized output $z_{n}(t)$ for $t=t_{0}$. If $t<t_{f}$ (where $t_{f}$ is a final time), then set $t=t_{0}+1$ and iterate the same procedures as the previous time $t$. If $t=t_{f}$, then stop here. The $n$ equalized outputs $z_{1}(t), \cdots, z_{n}(t)$ are obtained for $t=t_{0}, t_{0}+1, \cdots, t_{f}$.

Therefore, the proposed algorithm is summarized as shown in Table 1.

Table 1 . The proposed algorithm.

\section{Simulations}

To demonstrate the effectiveness of proposed method, some computer simulations were conducted. We considered an MIMO channel with two inputs and three outputs, and assumed that the length of channel is three $(K=3)$, that is 
$\boldsymbol{H}^{(k)} \mathrm{s}$ in (1) were set to be

$$
\begin{aligned}
& \boldsymbol{H}(z)=\sum_{k=0}^{2} \boldsymbol{H}^{(k)} z^{k}= \\
& {\left[\begin{array}{cc}
1.00+0.15 z+0.10 z^{2} & 0.65+0.25 z+0.15 z^{2} \\
0.50-0.10 z+0.20 z^{2} & 1.00+0.25 z+0.10 z^{2} \\
0.60+0.10 z+0.40 z^{2} & 0.10+0.20 z+0.10 z^{2}
\end{array}\right] .}
\end{aligned}
$$

The length of the equalizer was chosen to be seven $(L=7)$. We set the values of the tap coefficients to be zero expect for $w_{12}^{(4)}=w_{21}^{(4)}=1$. Two source signals were 4-PSK and 8PSK signals, respectively. For recovering first source signal, the initial values of $\tilde{\boldsymbol{R}}, \tilde{\boldsymbol{d}}_{i}$ and $\boldsymbol{P}$ were estimated using 5,000 data samples. For recovering second source signal, the initial value of $\tilde{\boldsymbol{R}}$ and $\boldsymbol{P}$ were set the identity matrix $\boldsymbol{I}$. The values of $\alpha$ and $\beta$ were chosen as $\alpha=0.999$ and $\beta=0.05$, respectively. Besides, we used the fourth-order correlation method for subtracting the contributions of an extracted source signal to the channel outputs. As a measure of performance, we use the multichannel intersymbol interference $\left(\mathrm{M}_{\mathrm{ISI}}\right)$ [3],[9].

Fig. 1 and Fig. 2 show performance results of our proposed algorithm for the time-invariant and the time-invariant channel, respectively, compared with the existing AMSEDA using the QR-factorization [10] with same conditions obtained by using 100,000 data samples. In Fig. 2, the last matrix $\boldsymbol{H}^{(2)}$ of the impulse response of the channel was varied by adding 0.3 to all its elements at discrete time $t=30,000$ for the proposed algorithm and at $t=2,500$ for the existing AMSEDA.

It can be seen from Fig. 1 that the proposed algorithm deconvolved all source signals and it is effective for the timeinvariant channel. Also it can be seen from Fig. 1 that in the proposed algorithm it takes much time (about $t=20,000$ ) until all source signals are deconvolved, however the accuracy is very high (about $-22 \mathrm{~dB}$ ) and the stability with the time is good. In the meantime, in the existing AMSEDA all source signals are deconvolved quickly (about $t=2,500$ ), however the accuracy is not so high (about $-3 \mathrm{~dB}$ ) and the stability with time is bad, because the value of $\mathrm{M}_{\mathrm{ISI}}$ gradually increases after all source signals are deconvolved.

It can be seen from Fig. 2 that the proposed algorithm is effective for even the time-variant channel, however the existing AMSEDA is not effective for the time-variant channel.

We think that one of reasons why the proposed algorithm is superior to the existing AMSEDA for the time-variant channel is that the pseudo-inversion lemma gives an explicit recursion formula for time-updating of calculating the pseudoinverse $\tilde{\boldsymbol{R}}^{\dagger}(t)$. This fact holds also true in the full-rank case of the correlation matrices.

\section{CONCLUSions}

We have considered the problem of adaptive multichannel blind deconvolution based on the super-exponential algorithms using deflation methods proposed by Inouye and Tanebe [3]. In this paper, we proposed a new approach to the adaptive multichannel deflationary blind deconvolution using the matrix pseudo-inversion lemma and the higher-order correlations. In order to see the effectiveness of the proposed approach, we

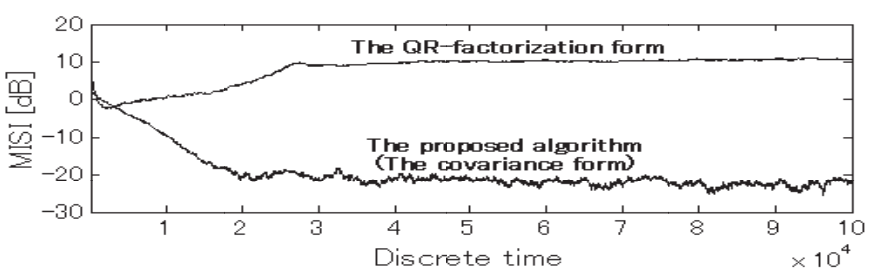

Fig. 1. Performance of the proposed algorithm for the non-adaptive model.

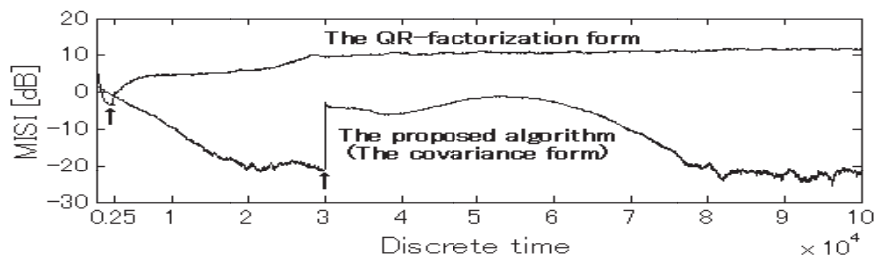

Fig. 2. Performance of the proposed algorithm for the adaptive model.

have considered computer simulations for two types of MIMO channels, that is, the first one is time-invariant and the second one is time-variant. It has been shown through computer simulations that the proposed approach is effective for timeinvariant channels and even time-variant channels.

\section{REFERENCES}

[1] Special issue on blind system identification and estimation, Proc. IEEE, vol. 86, no. 10, pp. 1907-2089, Oct. 1998.

[2] O. Shalvi and E. Weinstein, "Super-exponential methods for blind deconvolution," IEEE Trans. Information Theory, vol. 39, no. 2, pp. 504-519, Mar. 1993.

[3] Y. Inouye and K. Tanebe, "Super-exponential algorithms for multichannel blind deconvolution," IEEE Trans. Signal Processing, vol. 48, no. 3, pp. 881-888, Mar. 2000.

[4] M. Martone, "An adaptive algorithm for antenna array low-rank processing in cellular TDMA base stations," IEEE Trans. Commum., vol. 46, no. 5, pp. 627-643, May 1998.

[5] M, Martone, "Fast adaptive super-exponential multistage beamforming for cellular base-station transceivers with antenna arrays," IEEE Trans. Vehicular Tech., vol. 48, no. 4, Jul. 1999.

[6] K. L. Yeung and S. F. Yau, "A cumulant-based super-exponential algorithm for blind deconvolution of multi-input multi-output systems," Signal Process., vol. 67, pp. 141-162, 1998.

[7] M. Kawamoto, K. Kohno and Y. Inouye, "Robust Super-Exponential Methods for Deflationary Blind Equalization of Instantaneous Mixtures," to appear in IEEE Trans. Signal Processing.

[8] K. Kohno, Y. Inouye, M. Kawamoto and T. Okamoto, "Adaptive SuperExponential Algorithms for Blind Deconvolution of MIMO Systems," in Proc. ISCAS, vol.V, Vancouver, Canada, 2004, pp.680-683.

[9] K. Kohno, Y. Inouye and M. Kawamoto, "Super-Exponential Methods Incorporated with Higher-Order Correlations for Deflationary Blind Equalization of MIMO Linear Systems," in Proc. 5th Int. Conference on Independent Component Analysis and Blind Signal Separation (ICA2004), Granada, Spain, 2004, pp.685-693.

[10] K. Kohno, Y. Inouye, M. Kawamoto and T. Okamoto, "An Adaptive Super-Exponential Deflation Algorithm for Blind Deconvolution of MIMO Systems Using the QR-factorization of Matrix Algebra," in Proc. $M W S C A S$, vol.III, Hiroshima, Japan, 2004, pp.419-422.

[11] Y. Inouye and R-W. Liu, "A system-theoretic foundation for blind equalization of an FIR MIMO channel system," IEEE Trans. Circuits and Systems-I, Fundam. Theory Appl. vol. 49, no. 4, pp. 425436, Apr. 2002.

[12] G. H. Groub and C. F. Van Loan. Matrix Computations 2nd Ed., Baltimore, MD: The Johns Hopkins University Press, 1989

[13] S. Haykin. Adaptive Filter Theory 3rd Ed., Upper Saddle River, NJ: Prentice-Hall, 1996.

[14] G. W. Stewart, "Methods of simultaneous iteration for calculating eigenvectors of matrices," in Topics in Numerical Analysis II, J. H. Miller, Ed. New York: Academic, pp. 169-185, 1975. 